\title{
Allergic Fungal Sinusitis in Children
}

\section{Marco Berlucchi* and Barbara Pedruzzi}

Department of Pediatric Otorhinolaryngology, Spedali civili, Brescia, Italy

\begin{abstract}
Described first in 1980s, allergic fungal sinusitis that is a chronic noninvasive fungal sinusitis is a relatively uncommon disease in adults. Nasal polyposis, peculiar imaging features, and allergic mucin are its main characteristics. Although case report and/or series about this disease have been reported, its occurrence in pediatric population remains rare. Pathogenesis of this illness is the same in both children and adults, but some clinical characteristics are different, especially at presentation. The clinical picture and treatment of this disorder in children are analyzed.
\end{abstract}

Keywords: Allergic fungal sinusitis; Endoscopic sinus surgery; Pediatric sinusitis; Sinusitis

\section{Introduction}

Allergic fungal sinusitis (AFS) is a benign, extramucosal, noninvasive fungal sinus disease representing an allergic/hypersensitivity response to extramucosal fungal pathogens in the sinus [1]. It occurs mainly in immunocompetent patients, in contrast to invasive fungal diseases that usually involve an immunocompromised host. The incidence of AFS in patients affected by chronic rhinosinusitis and treated surgically is about 7\% [2-5]. However, this disorder is an underdiagnosed pathological entity [6], and some authors believe that only increased awareness among physicians to look for fungal involvement will increase the diagnosis of AFS.

In 1976, Safirstein first described AFS, noting that the combination of nasal polyposis, crust formation, and sinus cultures yielding Aspergillus was similar to that observed in allergic bronchopulmonary aspergillosis [7]. Five years later, chronic fungal sinusitis requiring surgery was described by Millar et al. [8]. In particular, 5 cases of chronic Aspergillus fumigates sinusitis were presented in which the sinus exudates appeared histologically similar to the bronchial mucus plugs observed in patients with allergic bronchopulmonary aspergillosis.

In 1983, Katzenstein et al. [2,9] described a new non-invasive form of chronic sinusitis seen in atopic individuals with nasal polyposis and intractable sinusitis. This disorder was termed "allergic aspergillus sinusitis" because it had features that were similar to allergic bronchopulmonary aspergillosis. Diagnosis was based on the following histological findings: (1) clumps or sheets of necrotic eosinophils; (2) Charcot-Leyden crystals (from degraded eosinophils); and (3) noninvasive fungal hyphae/fungal elements with morphology consistent with Aspergillus species within the nasal mucus.

In 1989, Robson et al. [3] introduced the term AFS since they identified several fungi causing the same disease. In 1994, in a large retrospective study by Cody et al. [5] reported that Aspergillus species were responsible for only about $15 \%$ of cases of AFS. In the same year, based on the clinical findings, Bent and Kuhn [10] proposed 5 criteria for the diagnosis of AFS: (1) nasal polyposis; (2) allergic mucin; (3) characteristic computed tomographic (CT) scan findings consistent with chronic rhinosinusitis; (4) positive fungal smear (histology or culture); and (5) type I hypersensitivity diagnosed by history, positive skin test, or serology. These criteria are now referred to as major criteria.

In 1995, deShazo and Swain [11] reported on 7 patients with AFS in whom Bent and Khun's criteria were applied with the exception of atopy. Atopy was excluded as a diagnostic criterion for AFS since after reviewing the literature only two-thirds of AFS-patients had a positive skin test result for fungi cultures. In 1998, Schubert and Goetz published the following histopathologic criteria for the diagnosis of AFS: 1) allergic mucin is present on gross and/or histopathologic evaluation; 2) either (a) methenamine silver stain of allergic mucin is positive for fungi but not fungal hyphae are seen in the mucosa or (b) silver stain is negative and fungal culture is positive; 3 ) sinus mucosal H\&E stain is characteristic for AFS and indistinguishable from the mucosal infiltrate in asthmatic bronchial mucosa; and 4) other histopathologic fungal disorders are excluded [12].

In 2003, Kuhn and Swain [13] described 5 major and 6 minor criteria for diagnosis of AFS in adults. The former were: (1) type I IgE-mediated hypersensitivity; (2) nasal polyposis; (3) characteristic CT findings; (4) allergic mucin; and (5) positive fungal smear and/ or culture. The latter criteria included: (1) asthma; (2) unilateral predominance; (3) radiographic bone erosion on CT; (4) positive fungal culture; (5) Charcot-Leyden crystals; and (6) serum eosinophilia. At present, these are the most widely accepted criteria for diagnosis of AFS. To resolve many controversies about chronic rhinosinusitis, the International Society for Human and Animal Mycology formed a working group on fungal sinusitis. In 2008 during a workshop in India, the group recommended using the term allergic fungal rhinosinusitis and eosinophilic mucin instead of allergic fungal sinusitis and allergic mucin, respectively. However, to date both terms are utilized in literature $[14,15]$.

In two large series by Cody et al. [5] and Bent et al. [16], the mean age of patients was 23 and 42 years, respectively with a female predominance in the adult population and a male prevalence in pediatric age $[1,17]$. However, some studies have described a similar gender predilection [18]. Furthermore, AFS is associated with nasal polyposis, asthma, and aspirin sensitivity in $100 \%, 65 \%$, and $27 \%$ of the cases, respectively $[5,12,19]$.

*Corresponding author: Marco Berlucchi, MD, Department of Pediatric Otorhinolaryngology, Spedali Civili, Piazza Spedali Civili 1, 25123 Brescia, Italy, Tel: +390303996226; Fax: +390303996009; E-mail: marco.berlucchi@tin.it

Received December 03, 2011; Accepted January 18, 2012; Published January 23, 2012

Citation: Berlucchi M, Pedruzzi B (2012) Allergic Fungal Sinusitis in Children. J Aller Ther S5:004. doi:10.4172/2155-6121.S5-004

Copyright: (c) 2012 Berlucchi M, et al. This is an open-access article distributed under the terms of the Creative Commons Attribution License, which permits unrestricted use, distribution, and reproduction in any medium, provided the original author and source are credited. 
The precise etiology is unknown. A hypersensitive response to extramucosal fungi in the paranasal sinuses is the most widely-accepted theory [6]. According to Gell and Coombs classification, a type I and III reaction has been postulated to play a role in the development of AFS. In atopic patients, the initial inflammatory response ensues as a result of both type I (IgE-mediated) and type III (immune complex mediated) reactions. The resulting tissue edema and obstruction of the sinus ostia lead to stasis of secretions within the sinuses. This situation is an ideal environment for further fungal proliferation, thereby increasing antigenic exposure [20]. Massive polyposis develops and fungal secretions distort sinus anatomy. It may then expand to involve other sinuses causing bony expansion and erosion [21]. However, this theory was questioned by Ponikau et al. [22]. In relation to their new observations, type I reaction in not important in the pathophysiology of AFS, but it is fundamental the role of eosinophils. For this reason, they proposed the term eosinophilic fungal sinusitis to identify this disorder. The most common fungal species responsible of AFS are Dematiaceous fungi including Bipolaris spicifera, Curvularia lunata, Alternaria, Exserohilum rostratum, Helminthosporium, Drechslera, Fusarium, Chrysosporium, Rhizopus [6,23]. These account for $84 \%$ of cases, followed in order of frequency by Aspergillus species (13\%) and other species $(<1 \%)$ [24]. Only rarely AFS is due to Trichoderma longibrachiatum [25].

The clinical features in patients with AFS may be similar to those of chronic sinusitis. Nasal obstruction, rhinorrhea, postnasal discharge, and/or headache are the main symptoms observed at presentation. Pain is uncommon in patients with AFS and when present suggests the concomitant presence of bacterial rhinosinusitis. Approximately onehalf of the patients with AFS have unilateral involvement and may show asymmetric involvement with facial disfigurement/dysmorphia such as proptosis, telecanthus and malar flattening $[12,26]$.

Nasal endosocopy can reveal nasal polyposis and allergic mucin. This latter feature is represented macroscopically by thick, tenacious, and highly viscous mucus [27].

Histologic examination of allergic mucin shows non-invasive fungal hyphae within sheets of eosinophils and Charcoat-Lyden crystals (Figure 1). Fontana Masson stains and Grocott-Gomori's methanamine silver stains can help identify the fungi responsible [19].

On non-contrast CT scan, patients with AFS frequently have soft tissue areas of attenuation with internal hyperdensity of the affected sinuses $[21,26,28]$. The allergic mucin contains fungal elements, which have a great affinity for calcium, manganese, magnesium and other ferromagnetic substances [29]. Bony erosion of the involved sinus walls due to the direct progression of the disease may be also evident (Figure 2). On MR, presence of hypointense/hypoattenuated/low central T1 signal, central T2 signal void, and increased peripheral T1/ T2 enhancement is highly specific for AFS [21]. The high protein and low water concentration of allergic mucin, coupled with the high water content within surrounding edematous paranasal sinus mucosa, gives rise to specific MR characteristics.

Diagnostic work-up for the patient with suspicious AFS also includes total eosinophil count, total serum IgE level, antigen specific IgE for both fungal and other inhalants, fungal antigen specific IgG, precipitating antibodies, microscopic evaluation and fungal culture of allergic mucin evacuated intraoperatively [18,29]. In 1999, Ponikau et al. [22] proposed new techniques for fungal detection in culture and histology. These diagnostic procedures were based on collecting a large amount of mucus, as much as possible, for culture and histologic

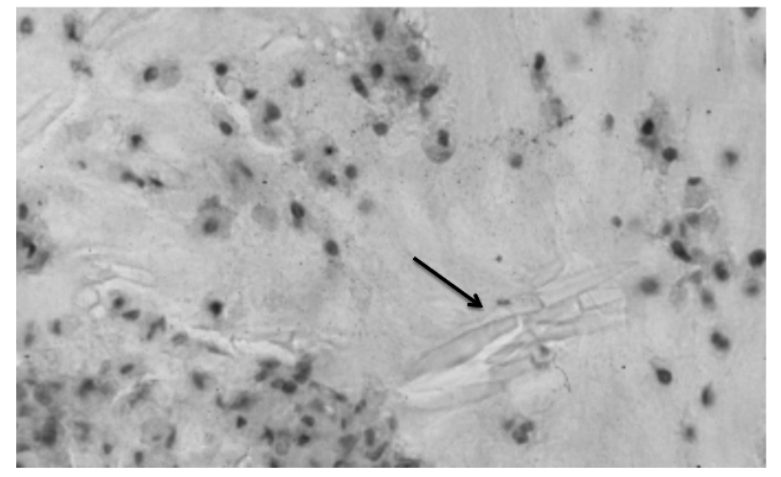

Figure 1: Charcoat-Lyden crystals (arrow) are observed at histologic examination of allergic mucin.

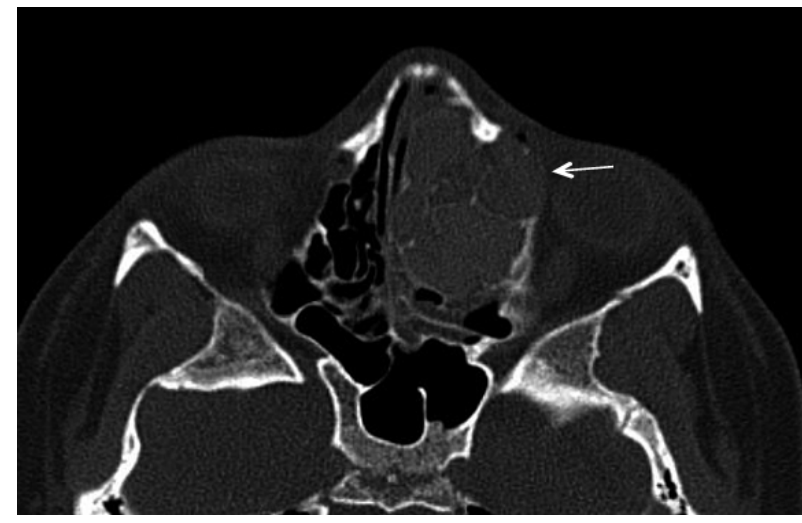

Figure 2: Axial CT scan showing a left ethmoid allergic fungal sinusitis with erosion of papyracea lamina (arrow).

examination, to increase the chances of isolating the pathogen. Multiple sections from different areas of the nose and paranasal cavities should be prepared as the fungi may be found scattered. Such techniques were utilized successfully by other researches [30]. In 2003, Dhiwakar et al. [31] reported that the triads including nasal polyps, characteristic CT scan findings, and specific IgE titers have a sensitivity of $70 \%$ and a specificity of $100 \%$ for preoperative diagnosis of AFS.

A wide consensus on the appropriate treatment of AFS has not been reached [32], and both medical and surgical procedures are used. Longterm medical therapy includes oral or topical corticosteroids, antifungal agents and/or immunotherapy. The anti-inflammatory and immunemodulated effects of systemic corticosteroids seem to control recurrence of disease in the postoperative period, but uniform data about dosage of drugs and length of therapy are not available. Moreover, several side effects in relation to their chronic use are well known. Preoperative use of steroids is helpful to reduce inflammation, mucosal edema, and polyps that can help to identify surgical landmarks [29]. Some authors recommend pre-operative use of steroids associated with antibiotics to reduce edema and the chances of superadded postoperative bacterial sinusitis, respectively $[29,33,34]$.

The use of antifungal agents for the treatment of AFS has shown variable results, and thus their role is controversial. Sullivan and Bent [24] reported that systemic antifungal therapy in AFS has no role since the fungal sinus disorder is extramucosal. In contrast, Khattar and Hathiram affirmed that oral itraconazole seems to be beneficial [29]. Finally, topical fluconazole nasal spray was used with positive results 
and, although there is not adequate evidence, some authors have performed nasal and paranasal amphotericin $\mathrm{B}$ washes to reduce the luminal load of the fungus, although there is not adequate evidence for its efficacy $[29,35]$.

Even if considered initially contraindicated because of perceived risk of a Gell and Coombs type III reaction due to antigens administration, immunotherapy is gaining an important role in treatment of AFS. In 1997, Mabry et al. [36] described their experience about administration of immunotherapy after surgery in a series of nine patients affected by AFS. The symptoms of their patients did not worsen and reformation of allergic mucin and nasal crust accumulation diminished [36]. These successful results were confirmed in further their trials $[37,38]$. In 2011, Bozeman et al. [32] reported good results with allergen immunotherapy to fungi to which the patient has specific IgE, and suggested that this treatment may be helpful in decreasing allergic inflammation and recurrence of AFS. Recently, Greenhaw et al. [39] evaluated the safety of high-dose subcutaneous fungal immunotherapy in 14 AFS-patients. No differences of numbers of immediate or delayed local reactions, or required dose adjustments were observed when the results were compared to those of the control group. Moreover, no patient showed evidence of immune complex disorder [39]. Removal of the fungal load from the paranasal sinuses is performed by surgery, and immunotherapy might achieve sufficient immune-modulation to benefit the patient [40]. Immunotherapy seems to reduce the need for systemic and topical corticosteroids and to reduce the incidence of recurrence $[37,41]$. However, additional trials are mandatory to support these results, as the long-term benefits are unknown.

From a surgical point of view, endoscopic sinus surgery is the standard surgical technique utilized in AFS-patients [42]. Removal of allergic mucin and nasal polyps, associated with opening of all the involved sinuses, is usually performed.

The natural course of AFS is characterized by frequent recurrence. Overall, recurrence rates range from $10-100 \%$ [43], and thus it is mandatory to perform close post-operative follow-up to identity and treat early recurrences. Herein, we reviewed the literature on AFS in pediatric population focusing on diagnosis and treatment.

\section{Pediatric Allergic Fungal Sinusitis}

Up to now, few reports about AFS in the pediatric population have been described $[1,17,20,27,44]$. As reported by McClay et al. [1], the clinical presentation and course of AFS in children is different than adults. In particular, pediatric AFS appears to be more aggressive. For this reason, early diagnosis, prompt management, and periodic postoperative endoscopic follow-ups are fundamental [45].

In the pediatric population, AFS is particularly frequent in male patients and usually affects adolescents $[17,46]$.

Children with AFS can be atopic, asthmatic, and present with nasal polyps. In 2006, Campbell et al. [27] found that their pediatric patients affected by AFS were atopic, have nasal polyposis, and that asthma was present in more than half of patients. Skin testing and/ or RAST results were positive for molds cultured from the sinuses, with a typical regional mold/fungal pattern. The following year, AlSwiahb et al. [17] found nasal polyposis in all their AFS-children that was associated with allergic rhinitis and bronchial asthma in $64 \%$ and $34 \%$ of patients, respectively. Moreover, skin tests were moldpositive. In contrast, another study found that there was a much higher likelihood of coexistent asthma and rhinobronchial allergy in adults than in children younger than 15 years $(50 \%$ vs $1 \%$, respectively)
[45]. These authors suggested that the previous data on AFS-children with underlying allergy were due to an insufficient quantity of reports comparing children and adults with AFS [45]. The fungi responsible for AFS in children are similar to those found in the adult population. In particular, Aspergillus, Curvularia, Alternaria, and Bipolaris spp were the predominant fungal species isolated $[1,17,27,45]$. No cases of histological fungal invasion into the orbital periostion and/or dura madre were found on pathological examinations [1].

Clinically, the main symptom in children with AFS is nasal obstruction $[17,44,45]$. The disorder is generally unilateral and facial asymmetry may be observed on presentation $[1,45]$. Owing to its aggressive regional extension, bone expansion, and erosion, it is possible to observe proptosis, telecanthus, malar flattening, nasal pyramid widening, ophthalmoplegia, and bulge in the medial canthus $[1,44,45]$.

Among these signs, proptosis at presentation is the most frequent facial deformity in pediatric patients [1,45]. In 2006, Gupta et al. [45] reported nasal obstruction and proptosis as the most common sign and symptom in children younger than 15 years. In the same year, Campbell et al. [27] confirmed that the presentation of AFS in children is associated with a high rate of proptosis. In 2007, Al-Swiahb et al. [17] reported proptosis in $20 \%$ of their patients, facial swelling in $12 \%$, and telecantus in $8 \%$. McClay et al. [1], who described a higher incidence of proptosis associated with or without telecanthus or malar flattening in pediatric population than in adults, hypothesized that facial deformities occur more frequently in children than adults because the expansible forces from AFS actively expand a more pliable bone. For this reason, AFS determines more dysmorphisms in the growing facial skeleton of the pediatric population than in the mature skeleton of adult patients [1]. In 2006, Campbell et al. [27] reported that proptosis was present in the $50 \%$ of their AFS-patients. These patients suffered also from recurrent sinonasal polyposis. Since this latter disease can promote proptosis, the correct etiology of this important ocular sign cannot be recognized [27].

Finally, Hermann et al. [47] described first case of irreversible unilateral visual loss in an immunocompetent child affected by AFS involving the sphenoid sinus. They suggested that the optic neuritis was due to venous congestion as a result of thrombophlebitis, and occlusion of the retinal artery following an increase in orbital pressure [47].

On imaging, the features of AFS in children are similar to those in adult patients. CT-scans showed heterogeneous soft tissue density mass in involved sinuses that appear expanded with patchy calcifications inside [45]. The mass with wispy areas of hyper-attenuation is a characteristic finding of AFS and is often associated with thinning of the sinus bony wall [17]. On imaging, bony erosion of the sinus walls and extension of the pathology into nearby sites occur at a similar rate in children and adults, ranging from $20-50 \%[1,27]$. At MR, a low T1weighted signal and signal void on T2-weighted sequences are typical findings of AFS (Figure 3,4). This latter signal gives the impression that the disorder disappears on imaging.

Treatment of AFS in pediatric cases does not differ from that in adults, and endoscopic sinus surgery is an important therapeutic step. Surgical debulking of diseased tissue, removal of fungal debris from involved sinuses, and establishment of normal mucociliary drainage pathways are the main goals. The routine use of intraoperative saline irrigations allows removal of fungal impaction and allergic mucin. Moreover, even if no definitive data have been described, intraoperative use of amphotericin B or ketoconazole dissolved in saline solution 


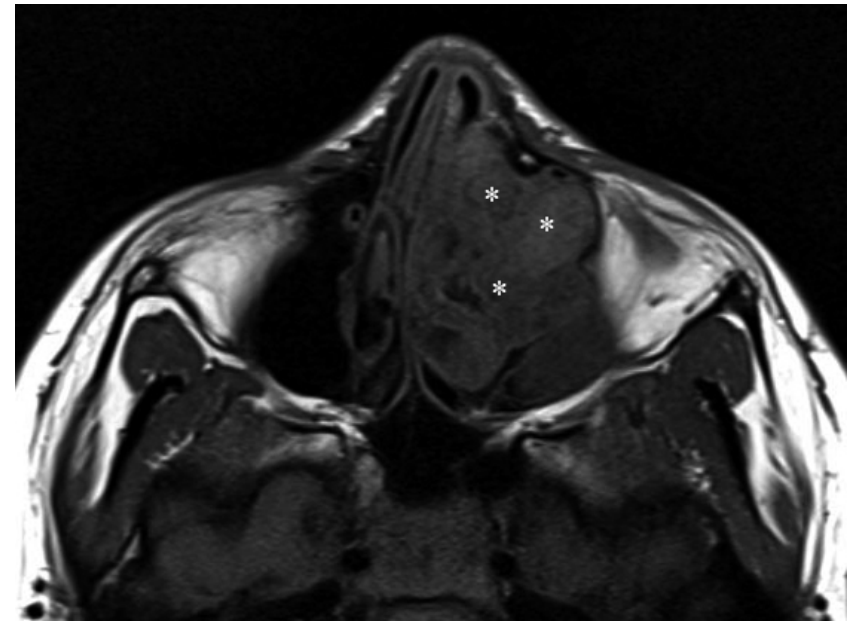

Figure 3: Axial MR revealing a hypointense signal (asterisks) on T1-weighted sequence.

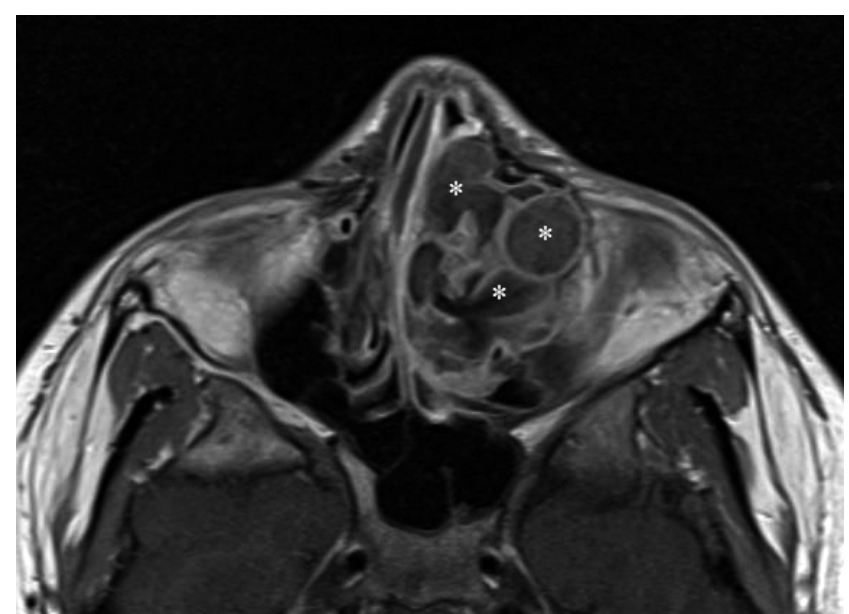

Figure 4: Axial MR displaying several signal voids (asterisks) on T2-weighted sequence.

or acetic acid, respectively, as nasal irrigations could have beneficial effects $[27,46]$. Because of anatomical modifications, during surgical intervention the surgeon must be aware of the high risk of penetration into anterior cranial fossa and/or orbit [46].

In the post-operative period, saline nasal irrigation, nasal corticosteroid sprays, and oral steroids are administrated to avoid recurrence [27]. However, systemic corticosteroids should be stopped as soon as possible to avoid adverse effects, especially on growth and bone development [46]. Antifungal nasal agents such as fluconazole seem to be useful in preventing progression of disease and/or improving symptoms, but to date limited data is available [27].

In addition to medical treatments, endoscopic cleanings should be periodically scheduled after surgery, and patients should be followed for a very long period [46]. Finally, as described in the study by Campbell et al. [27], allergen immunotherapy may have a therapeutic role. Although this treatment was well tolerated, definite efficacy has not been documented in all treated cases. This therapy may be used as adjuvant therapy and utilized after removal of a considerable amount of the fungal disease from sinuses.
The rate of relapse in pediatric cases ranges from 25 to $55 \%$, and is higher than in adults $[17,27,45]$. It has been hypothesized that this high rate of recurrence in the pediatric population is related to the risks associated with long-term or repetitive systemic steroid therapy [27] However, patients with recurrence usually undergo surgical revision $[17,45]$.

\section{Conclusions}

Pediatric AFS is an uncommon disorder respect to adult one. Pathogenesis of this disease and the fungal pathogens responsible for AFS in children are similar to adult cases, although clinical presentation is different. In particular, asymmetrical disorders and facial anomalies such as proptosis are more frequent in children than adults. Imaging is mandatory before surgical treatment, and therapy is similar in both children and adults. Surgical debridement of the lesion and cleaning of involved sinuses by an endoscopic sinus approach is the gold standard of treatment. Administration of topical and/or systemic steroids plays an important role in prevention of recurrence. However, the prolonged use of systemic steroids may favor negative effects on growth and development of pediatric patients. Finally, the surgeon must keep this in mind and closely follow patients for early diagnosis and prompt, adequate therapy. Endoscopic follow-ups are mandatory to identify and treat recurrence.

\section{References}

1. McClay JE, Marple B, Kapadia L, Biavati MJ, Nussenbaum B, et al. (2002) Clinical presentation of allergic fungal sinusitis in children. Laryngoscope 112 565-569.

2. Katzenstein AL, Sale SR, Greenberger PA (1983) Allergic Aspergillus sinusitis: a newly recognized form of sinusitis. J Allergy Clin Immunol 72: 89-93.

3. Robson JM, Hogan PG, Benn RA, Gatenby PA (1989) Allergic fungal sinusitis presenting as a paranasal sinus tumour. Aust N Z J Med 19: 351-353.

4. Ence BK, Gourley DS, Jorgensen NL, Shagets FW, Parsons DS (1990) Allergic fungal sinusitis. Am J Rhinol 4: 169-178.

5. Cody DT, Neel HB, Ferreiro JA, Roberts GD (1994) Allergic fungal sinusitis: the Mayo Clinic experience. Laryngoscope 104: 1074-1079.

6. Schubert MS (2000) Medical treatment of allergic fungal sinusitis. Ann Allergy Asthma Immunol 85: 90-97.

7. Safirstein BH (1976) Allergic bronchopulmonary aspergillosis with obstruction of the upper respiratory tract. Chest 70: 788-790.

8. Millar JW, Johnston A, Lamb D (1981) Allergic aspergillosis of the maxillary sinuses. Thorax 36: 710 .

9. Katzenstein AL, Sale SR, Greenberger PA (1983) Pathologic findings in allergic Aspergillus sinusitis: a newly recognized form of sinusitis. Am J Surg Pathol 7 439-443.

10. Bent JP, Kuhn FA (1994) Diagnosis of allergic fungal sinusitis. Otolaryngo Head Neck Surg 111: 580-588.

11. deShazo RD, Swain RE (1995) Diagnostic criteria for allergic fungal sinusitis. J Allergy Clin Immunol 96: 24-35.

12. Schubert MS, Goetz DW (1998) Evaulation and treatment of allergic funga sinusitis. I. Demographics and diagnosis. J Allergy Clin 102: 387-394.

13. Kuhn FA, Swain R (2003) Allergic fungal sinusitis: diagnosis and treatment Curr Opin Otolaryngol Head Neck Surg 11: 1-5.

14. Schubert MS (2009) Allergic fungal sinusitis: pathophysiology, diagnosis and management. Med Mycol 47 (Suppl 1): S324-330.

15. Glass D, Amedee RG (2011) Fall Allergic fungal rhinosinusitis: a review. Ochsner J 11: 271-275

16. Bent JP, Kuhn FA (1996) Antifungal activity against allergic fungal sinusitis organisms. Laryngoscope 106: 1331-1334. 
17. Al-Swiahb JN, Al-Ammar A, Al-Dousary SH (2007) Allergic fungal sinusitis in children in Saudi Arabia. Saudi Med J 28: 1711-1714

18. Houser SM, Corey JP (2000) Allergic fungal rhinosinusitis: pathophysiology, epidemiology, and diagnosis. Otolaryngol Clin North Am 33: 399-409.

19. Morpeth JF, Rupp NT, Dolen WK, Bent JP, Kuhn FA (1996) Fungal sinusitis: an update. Ann Allergy Asthma Immunol 76: 128-139.

20. Manning SC, Vuitch F, Weinberg AG, Brown OE (1989) Allergic aspergillosis: a newly recognized form of sinusitis in the pediatric population. Laryngoscope 99: 681-685.

21. Manning SC, Merkel M, Kriesel K, Vuitch F, Marple B (1997) Computed tomography and magnetic resonance diagnosis of allergic fungal sinusitis. Laryngoscope 107: 170-176.

22. Ponikau JU, Sherris DA, Kern EB, Homburger HA, Frigas E, et al. (1999) The Diagnosis and Incidence of allergic fungal sinusitis. Mayo Clin Proc 74: 877884.

23. Manning SC, Schaefer SD, Close LG, Vuitch F (1991) Culture-positive allergic fungal sinusitis. Arch Otolaryngol Head Neck Surg 117: 174-178.

24. Sullivan DP, Bent JP (2001) Allergic Fungal Sinusitis: Diagnosis and Management. Op Tech Otolaryngol Head Neck Surg 12: 2-6.

25. Tang P, Mohan S, Sigler L, Witterich I, Summerbell R, et al. (2003) Allergic fungal sinusitis associated with Trichoderma longibrachiatum. J Clin Microbiol 41: $5333-5336$.

26. Mukherji SK, Figueroa RE, Ginsberg LE, Zeifer BA, Marple BF, et al. (1998) Allergic fungal sinusitis: CT findings. Radiology 207: 417-422.

27. Campbell JM, Graham JM, Gray HC, Bower C, Blaiss MS, et al. (2006) Allergic fungal sinusitis in children. Ann Allergy Asthma Immunol 96: 286-290.

28. Zinreich SJ, Kennedy DW, Malat J, Curtin HD, Epstein JI, et al. (1988) Fungal sinusitis: diagnosis with CT and MR imaging. Radiology 169: 439-444.

29. Khattar VS, Hathiram BT (2009) Allergic fungal Rhinosinusitis. Otorhinolaryngo Clin Int J 1: 37-44.

30. Braun $\mathrm{H}$, Stammberger $\mathrm{H}$, Buzina W, Freudenschuss $\mathrm{K}$, Lackner A, et al (2003) Incidence and detection of fungi and eosinophilic granulocytes in chronic rhinosinusitis. Laryngorhinootologie 82: 330-340.

31. Dhiwakar M, Thakar A, Bahadur S, Sarkar C, Banerji U, et al. (2003) Preoperative diagnosis of allergic fungal sinusitis. Laryngoscope 113: 688-694

32. Bozeman S, deShazo R, Stringer S, Wright $L$ (2011) Complications of allergic fungal sinusitis. Am J Med 124: 359-368.
33. Marple BF, Mabry RL (1998) Comprehensive management of allergic fungal sinusitis. Am J Rhinol 12: 263-268.

34. Marple BF (2000) Allergic fungal rhinosinusitis. Surgical Management Otolaryngol Clin North Am 33: 409-418.

35. Jen A, Kacker A, Huang C, Anand V (2004) Fluconazole nasal spray in the treatment of allergic fungal sinusitis: a pilot study. Ear Nose Throat J 692: 694 695.

36. Mabry RL, Manning SC, Mabry CS (1997) Immunotherapy in the treatment of allergic fungal sinusitis. Otolaryngol Head Neck Surg 116: 31-35.

37. Mabry RL, Mabry CS (1997) Immunotherapy for allergic fungal sinusitis: the second year. Otolaryngol Head Neck Surg 117: 367-371.

38. Mabry RL, Marple BF, Folker RJ, Mabry CS (1998) Immunotherapy for allergic fungal sinusitis: three years' experience. Otolaryngol Head Neck Surg 119 648-651.

39. Greenhaw B, deShazo RD, Arnold J, Wright L (2011) Fungal immunotherapy in patients with allergic fungal sinusitis. Ann Allergy Asthma Immunol 107: 432436.

40. Mabry RL, Mabry CS (2000) Allergic fungal rhinosinusitis: the role of immunotherapy. Otolaryngol Clin North Am 33: 433-440.

41. Mabry RL, Marple BF, Mabry CS (2000) Outcomes after discontinuing immunotherapy for allergic fungal sinusitis. Otolaryngol Head Neck Surg 122 104-106.

42. Roth M (1994) Should oral steroids be the primary treatment for allergic funga sinusitis? Ear Nose Throat J 73: 928-930.

43. Marple BF (2001) Allergic fungal rhinosinusitis: current theories and management strategies. Laryngoscope 111: 1006-1019.

44. Gupta AK, Ghosh AK, Gupta AK (2003) Sinonasal aspergillosis in immunocompetent Indian children: an eight-year experience. Mycoses 46 455-461.

45. Gupta AK, Bansal S, Gupta A, Mathur N (2006) Is fungal infestation of paranasa sinuses more aggressive in pediatric population? Int J Pediatr Otorhinolaryngo 70: 603-608.

46. Kupferberg SB, Bent JP (1996) Allergic fungal sinusitis in the pediatric population. Arch Otolaryngol Head Neck Surg 122: 1381-1384.

47. Herrmann BW, White FV, Forsen JW (2006) Visual loss in a child due to allergic fungal sinusitis of the sphenoid. Otolaryngol Head Neck Surg 135: 328-329.
This article was originally published in a special issue, Allergic Rhinosinusitis handled by Editor(s). Reginald F. Baugh, University of Toledo, USA. 